\title{
Self-Assembled Dextrin Nanogel as Curcumin Delivery System
}

\author{
Catarina Gonçalves ${ }^{1}$, Paula Pereira ${ }^{1}$, Peter Schellenberg ${ }^{2}$, Paulo J. Coutinho ${ }^{2}$, Francisco M. Gama ${ }^{{ }^{*}}$ \\ ${ }^{1}$ Institute for Biotechnology and Bioengineering (IBB), Centre for Biological Engineering, Campus de Gualtar, University of Minho, \\ Braga, Portugal; ${ }^{2}$ Centre of Physics, Campus Gualtar, University of Minho, Braga, Portugal. \\ Email: fmgama@deb.uminho.pt
}

Received January $14^{\text {th }}, 2012$; revised February $24^{\text {th }}, 2012$; accepted March $15^{\text {th }}, 2012$

\begin{abstract}
Curcumin is a natural polyphenol with anti-oxidative, anti-inflammatory and anti-cancer properties. Its therapeutic potential is substantially hindered by the rather low water solubility and bioavailability, hence the need for suitable carriers. In this study, we show that self-assembled nanogels obtained from hydrophobically modified dextrin are effective curcumin nanocarriers. The stability and loading efficiency of curcumin-loaded nanogel depends on the nanogel/curcumin ratio. Higher stability of the formulation is achieved in water than in PBS buffer, as evaluated by dynamic light scattering and fluorescence measurements. The in vitro release profile, using sink conditions, indicates that dextrin nanogel may perform as a suitable carrier for the controlled release of curcumin. Biological activity of curcumin-loaded nanogel in HeLa cell cultures was assessed using the MTS assay.
\end{abstract}

Keywords: Curcumin; Dextrin; Nanogel; Drug Delivery; Cytotoxicity

\section{Introduction}

Curcumin is a multi-functional and pharmacologically safe natural agent. Chemically it is a low-molecularweight polyphenol derivative, extracted from rhizomes of Curcuma species. Used as a food additive for centuries, it has been recently demonstrated that curcumin is highly pleiotropic, interacting physically with diverse molecular targets, which includes transcription factors, growth factors and their receptors, cytokines, enzymes, and genes regulating cell proliferation and apoptosis [1]. Curcumin possess potent anti-inflammatory, anti-tumor and antioxidative (free radical scavenging activity) properties. Pre-clinical data shows that curcumin inhibits the formation of tumors in animal models of carcinogenesis, induce apoptosis in cancer cells of different tissues or organs, such as colon, breast, prostate and lung, acting on a variety of signal transduction pathways and molecular targets involved in the development of cancer [2]. The ability of curcumin to induce apoptosis in cancer cells, without cytotoxic effects on the healthy ones, is suggestive of a relevant anti-cancer potential. For instance, curcumin leads to apoptosis in scleroderma lung fibroblasts without affecting normal lung fibroblasts [3]. Preferential uptake of curcumin by tumor cells compared to normal cells was also demonstrated.

Curcumin, being a lipophilic molecule, interacts with *Corresponding author. the cellular membrane and is subsequently internalized, probably by diffusion. The higher curcumin uptake by tumor cells, against normal ones, has been assigned to various hypothetic factors, including the different membrane structure, protein composition and larger size [4]. The anti-oxidant activity of curcumin has been identified as the key mechanism by which this dietary phytochemical prevents cancer in vivo. Curcumin also allows reduction of oxidative and inflammatory stress in Alzheimer patients [5]. Indeed, curcumin has also been shown to inhibit mediators of inflammation such as cyclo-oxygenase-2 and lipooxygenase (two enzymes involved in inflammation) [6], inducible nitric oxide synthase (enzyme that catalyzes the production of nitric oxide) [7], cytokines and NFKB.

Despite all these promising characteristics, a major problem with curcumin is the very low solubility in aqueous solutions, which limits bioavailability and clinical efficacy. Interest in the development of nanocarriers for curcumin therapy is emerging. Nanogels, also called macromolecular micelles, have gained attention due to several advantages, such as low toxicity, high stability and small size, which may allow passive targeting of solid tumor tissues by enhanced permeation and retention (EPR) effect. Being highly hydrophobic, curcumin is insoluble in water but soluble in ethanol, dimethylsulfoxide or acetone. The encapsulation of a highly hydrophobic 
compound like curcumin in nanocarriers makes it readily soluble in aqueous systems, improving the ease of dosing and making intravenous administration possible. In a clinical study, after oral administration of $2 \mathrm{~g} \cdot \mathrm{kg}^{-1}$ of curcumin to rats, a maximum serum concentration of (1.35 \pm $0.23) \mu \mathrm{g} \cdot \mathrm{mL}^{-1}$ was observed at time $0.83 \mathrm{~h}$, whereas in humans the same dose of curcumin resulted in extremely low $\left((0.006 \pm 0.005) \mu \mathrm{g} \cdot \mathrm{mL}^{-1}\right.$ at $\left.1 \mathrm{~h}\right)$ serum levels [8].

In a previous work, we described the production and characterization of new nanogels, obtained by self-assembling of hydrophobically modified dextrin. The use of this material for curcumin solubilisation and delivery is now reported. Curcumin loading efficiency, stability and release profile were studied. The nanogel biocompatibility and the bioactivity of the curcumin-loaded nanogel formulation were evaluated using the $(3-(4,5-$ dimethylthiazol-2-yl)-5-(3-carboxymethoxyphenyl)-2-(4sulfophenyl)-2H-tetrazolium) (MTS assay) and HeLa cell cultures.

\section{Methods}

\subsection{Materials}

Dextrin-VA-SC $\mathrm{SC}_{16}\left(\mathrm{dex}_{16}\right)$ was synthesized as comprehensively described previously [9]. DexC $\mathrm{C}_{16}$ is composed by a hydrophilic dextrin backbone with grafted acrylate groups (VA — vinyl acrylate), which are partially substituted with long alkyl chains $\left(\mathrm{SC}_{16}\right)$. In this work, $\operatorname{dex} \mathrm{C}_{16}$ with a dextrin degree of substitution, with acrylate groups and alkyl chains of $13.0 \%\left(\mathrm{DS}_{\mathrm{VA}}\right)$ and $6.0 \%$ $\left(\mathrm{DS}_{\mathrm{C} 16}\right)$ respectively, was used. Curcumin was purchased from Fluka and (3-(4,5-dimethylthiazol-2-yl)-5-(3-carboxymethoxyphenyl)-2-(4-sulfophenyl)-2H-tetrazolium) (MTS) from Promega. SAMSA (5-((2-(and-3)-S-(acetylmercapto)succinoyl) amino) fluorescein (SAMSA-F) was obtained from Invitrogen. Ethanol was purchased from AppliChem.

\subsection{Nanogel Preparation}

Nanogel was obtained by dispersion of $\operatorname{dex} \mathrm{C}_{16}$ (lyophilized powder) in water, or phosphate-buffered saline (PBS) solution, under stirring at $50^{\circ} \mathrm{C}$, until a clear solution was obtained. This procedure avoids the use of any organic solvent. The dispersion was then filtrated through a $0.22 \mu \mathrm{m}$ syringe filter. The polymer concentration must be higher than $0.008 \mathrm{mg} / \mathrm{mL}$, previously identified as the critical micelle concentration (cmc) [9] required for the amphiphilic polymer to self-assemble in water, originating nanostructures containing hydrophobic domains, allowing the solubilization of hydrophobic molecules.

\subsection{Nanogel Stability}

The stability of dextrin nanogel $\left(\mathrm{DS}_{\mathrm{C} 16} 6.0 \%\right)$, in water or
PBS solution, was evaluated analysing the nanogel size distribution, up to 12 days. The size distribution was determined by dynamic light scattering (DLS) using a Malvern Zetasizer, MODEL NANO ZS (Malvern Instruments Limited, UK). The nanogel dispersion was analysed at $25^{\circ} \mathrm{C}$ in a polystyrene cell, using a $\mathrm{He}-\mathrm{Ne}$ laser-wavelength of $633 \mathrm{~nm}$ and a detector angle of $173^{\circ}$. The DLS analysis provides the characterization of a sample through the mean value (z-average) for the diameter, and a width parameter known as the polydispersity index (PdI).

\subsection{SAMSA-F Labeled Nanogel}

In order to label nanogel with SAMSA fluorescein (SAMSA-F), the following solutions were prepared: (1) Nanogel solution-10 mg of $\operatorname{dex}_{16}$ were dissolved in $1.3 \mathrm{~mL}$ of sodium phosphate buffer $0.1 \mathrm{M} \mathrm{pH} 7$ and stirred for $30 \mathrm{~min}$; (2) fluorescein solution-5 $\mathrm{mg}$ of SAMSA-F were dissolved in $0.5 \mathrm{~mL}$ of $\mathrm{NaOH} 0.1 \mathrm{M}$ and stirred for $15 \mathrm{~min}$. Afterwards, $7 \mu \mathrm{L}$ of $\mathrm{HCl} 6 \mathrm{M}$ and 0.1 $\mathrm{mL}$ of $\mathrm{NaPO}_{4}$ buffer $0.5 \mathrm{M} \mathrm{pH} 7$ were added and stirred for $10 \mathrm{~min}$. Finally, these two solutions were mixed up and stirred for $30 \mathrm{~min}$. Unbound fluorescein was separated using gel filtration in a sephadex G25 PD10 column pre-equilibrated. SAMSA-F labeled nanogel was eluted with PBS or distilled water, sterilized by filtration through a $0.22 \mu \mathrm{m}$ syringe filter and stored at $4{ }^{\circ} \mathrm{C}$. SAMSA-F labelling did not affect the nanogel size distribution, as evaluated by dynamic light scattering.

\subsection{Fluorescence Measurements}

Fluorescence studies were performed with SAMSA-F labeled nanogel $(0.115 \mathrm{mg} / \mathrm{mL})$ dispersed in distilled water or PBS, over time, using time resolved fluorescence to ascertain the occurrence of structural modifications. The fluorescence decay times were determined using the frequency-doubled output of a $\mathrm{Ti}: \mathrm{Sa}$ laser at $399 \mathrm{~nm}$ for excitation and measuring the emission at 540 nm. The decay curves were gained by Time Correlated Single Photon Counting (TCSPC) using a Becker \& Hickl SPC-150 computer board collecting the photon detection events from a Hamamatsu MCP R3809U-51 photon counting unit. The time resolution was $20 \mathrm{ps}$.

\subsection{Curcumin Loading, Efficiency and Stability}

Curcumin has been loaded into the hydrophobic domains of dextrin nanogel dispersed in distilled water or PBS. The physical entrapment of curcumin into the nanogel was performed following the nanogel preparation procedure, as described ahead. A stock solution of curcumin in ethanol with a concentration of $2.7 \mathrm{mM}$ was prepared. The required volume of curcumin from this solution was 
added to the nanogel dispersion (final concentration of ethanol $<1 \%$ ). The effect of nanogel/curcumin ratio on the loading efficiency and stability of the formulation was studied. Different formulations were prepared, using distilled water or PBS as dispersion medium, by varying polymer $(0.1,0.25,1.0$ or $2.0 \mathrm{mg} / \mathrm{mL})$ and the curcumin $(10,30,50 \mu \mathrm{M})$ concentrations. In order to evaluate the stability of the dispersions, they were kept under stirring at $37^{\circ} \mathrm{C}$ up to 8 days. At pre-defined periods of time, they were centrifuged at $4000 \mathrm{rpm}$ (Sigma 4K-15 refrigerated centrifuge) and $4^{\circ} \mathrm{C}$, for $10 \mathrm{~min}$, to remove the insoluble curcumin. The clear yellowish supernatant was carefully collected and analyzed spectrophotometrically. The quantification was carried out using a calibration plot obtained with curcumin concentrations between 0.3 and $50 \mu \mathrm{M}$. The loading efficiency was calculated by the following equation:

$$
\text { Loading efficiency }(\%)=\frac{[\mathrm{CM}]_{\text {loaded }}}{[\mathrm{CM}]_{\text {added }}} \times 100
$$

\subsection{Curcumin Release}

In the present work, the release of curcumin from the nanogel was studied using sink conditions (sample in a dialysis membrane) and a closed system (sample in a flask). The curcumin-loaded nanogel (stable formulation: $30 \mu \mathrm{M}$ of curcumin and $1.0 \mathrm{mg} / \mathrm{mL}$ of polymer dispersed in distilled water) was kept in a glass flask or transferred to a dialysis bag. The dialysis bag (molecular cut off of $10 \mathrm{kDa}$ ) containing the formulation was placed in 200 $\mathrm{mL}$ of distilled water or PBS, shaken under $500 \mathrm{rpm}$ at $37^{\circ} \mathrm{C}$. At predefined periods (up to $24 \mathrm{~h}$ ), a sample of 200 $\mu \mathrm{L}$ was withdrawn from each condition, centrifuged to guarantee removal of unload curcumin and soluble curcumin (supernatant) was quantified spectrophotometrically (Jasco V560). At the end of the assay, after $24 \mathrm{~h}$, the size distribution of the nanogel was evaluated by DLS.

\subsection{Cell Culture and Cytotoxicity Assay}

The cytotoxicity of unload or loaded curcumin was evaluated by MTS, a colorimetric assay that gives a measure of the mitochondrial metabolic activity. Human cervical cancer cells (HeLa) were counted and seeded at $1 \times 10^{4}$ cells/well into 24 -well cell culture plates, in cDMEM medium with $10 \%$ fetal bovine serum, and allowed to adhere for $24 \mathrm{~h}$ at $37^{\circ} \mathrm{C}$ and $5 \% \mathrm{CO}_{2}$ atmosphere. Afterwards, the culture medium was decanted and replaced with fresh culture medium or supplemented with nanogel $(0.1$ or $0.25 \mathrm{mg} / \mathrm{mL})$, unload curcumin (30 $\mu \mathrm{M}$ in ethanol) or curcumin-loaded nanogel (in distilled water). Then, plates were incubated for 24 or $48 \mathrm{~h}$ in triplicate, at $37^{\circ} \mathrm{C}, 5 \% \mathrm{CO}_{2}$. The cell viability after treat- ment was determined by the MTS assay. The culture medium of each well was decanted and replaced with $100 \mu \mathrm{L}$ of fresh culture medium. Then, $20 \mu \mathrm{L}$ of "CellTiter $96^{\circledR}$ AQueous One Solution Reagent" was added into each well and the plates were incubated for $2 \mathrm{~h}$ at $37^{\circ} \mathrm{C}, 5 \% \mathrm{CO}_{2}$. The amount of soluble formazan produced by cellular reduction of the MTS was measured at $490 \mathrm{~nm}$. The absorbance at $490 \mathrm{~nm}$ is proportional to the cell number.

\section{Results}

\subsection{Nanogel Stability}

In this work, the size stability of dextrin nanogel $\left(\mathrm{DS}_{\mathrm{C} 16}\right.$ $6.0 \%$ ), dispersed in distilled water or PBS solution, was evaluated (Table 1), up to 12 days. The nanogel prepared in a saline solution (buffer) present a gradual size increase along with the incubation period, apparently associated to a swelling process.

\subsection{Curcumin Loading, Efficiency and Stability}

The curcumin loading into the nanogel was analysed. Soluble curcumin, dissolved in a suitable solvent such as ethanol (Figure 1) exhibits intense absorption in the wavelength range from 350 to $550 \mathrm{~nm}$, with a maximum absorption at $428 \mathrm{~nm}$. The addition of curcumin dissolved in ethanol to water (to a final concentration of ethanol $<1 \%$ ), leads to curcumin precipitation. On the other hand, the addition of curcumin to aqueous dispersions of nanogel results in a bright yellow solution, suggesting the loading of curcumin, presumably into the hydrophobic domains within the nanogel. Indeed, the UV-Vis spectra reveal an intense absorption at $428 \mathrm{~nm}$, confirming the curcumin dissolution (Figure 1).

The size distribution of empty or curcumin-loaded dextrin nanogel (dispersed in distilled water) was determined by dynamic light scattering. The mean diameters (z-values) were found to be $61.1 \mathrm{~nm}$ (PdI 0.243) and 74.6 nm (PdI 0.368), respectively.

The stability of the curcumin-loaded nanogel, dispersed in distilled water or PBS, was evaluated spectro-

Table 1. Evaluation of mean diameter (z-avg) and polydispersity index (PdI), of dextrin nanogel $\left(\mathrm{DS}_{\mathrm{C} 16} 6.0 \%\right)$ dispersed in distilled water or PBS solution, up to 12 days.

\begin{tabular}{ccccc}
\hline & \multicolumn{2}{c}{ Water } & \multicolumn{2}{c}{ PBS solution } \\
\hline time & z-avg $(\mathrm{nm})$ & $P d I$ & z-avg $(\mathrm{nm})$ & $P d I$ \\
freshly prepared & 61.1 & 0.243 & 59.2 & 0.290 \\
3 days & 62.3 & 0.304 & 72.2 & 0.312 \\
5 days & 61.0 & 0.270 & 79.1 & 0.285 \\
12 days & 58.7 & 0.244 & 100.0 & 0.247 \\
\hline
\end{tabular}




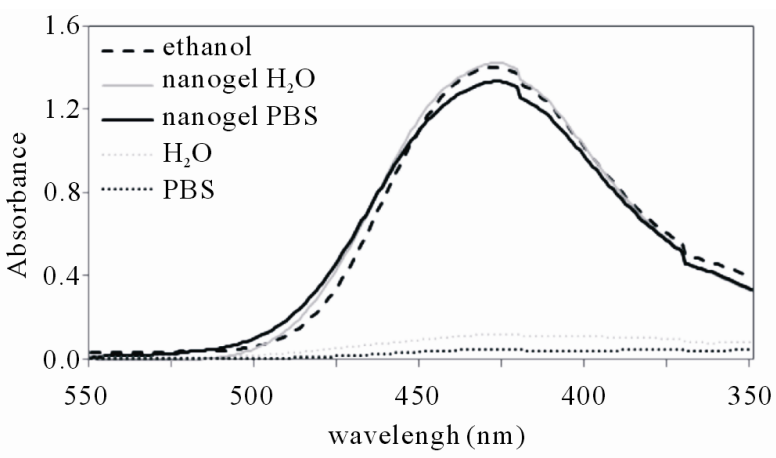

Figure 1. Ultraviolet-visible absorbance spectra of curcumin supernatant's dissolved in ethanol, aqueous nanogel dispersion, PBS nanogel dispersion, distilled water or PBS solution.

photometrically, using different formulations (ratio nanogel/curcumin). Figure 2 shows the concentration of soluble (encapsulated) curcumin obtained using different conditions, namely varying the incubation time, the initial concentration of curcumin $(10,30,50 \mu \mathrm{M})$ and nanogel $(0.1,0.25,1.0,2.0 \mathrm{mg} / \mathrm{mL})$, in both distilled water (Figure 2(a)) or PBS (Figure 2(b)).

The loading efficiency and stability are significantly affected by the nanogel/curcumin ratio used. High loading efficiency of curcumin is achieved, in the first period of observation (3h), when higher polymer concentrations are used. For example, $2.0 \mathrm{mg} / \mathrm{mL}$ allows almost 100\% loading efficiency for every curcumin concentrations studied, at $3 \mathrm{~h}$ after incorporation.

Regarding curcumin stability, a relevant difference is noticeable for longer incubation time frames: curcumin solutions are unstable for every formulations studied when PBS is used as dispersiom medium (Figure 2(b)).

In aqueous medium, for low nanogel concentrations, the stability is compromised when higher amounts of curcumin are used. However, high stability is achieved for higher nanogel concentrations.

SAMSA-F was used to further analyse the nanogel structural changes observed with aging time. Figure 3 shows the SAMSA-F fluorescence decays for nanogel dispersed in distilled water or in PBS over time, up to 14 days. We can see that there is a systematic trend of faster decay of SAMSA-F with aging time, in the case of nanogel dispersed in PBS.

\subsection{Curcumin Release}

To ascertain the suitability of dextrin nanogel as delivery system for hydrophobic drugs such as curcumin, the in vitro release was studied. Two different methods were used to evaluate the release profile of curcumin-loaded nanogel. Loaded nanogel was kept in open (sink conditions, dialysis membranes) or closed (flask) systems. At pre-defined time intervals, a sample was withdrawn (in

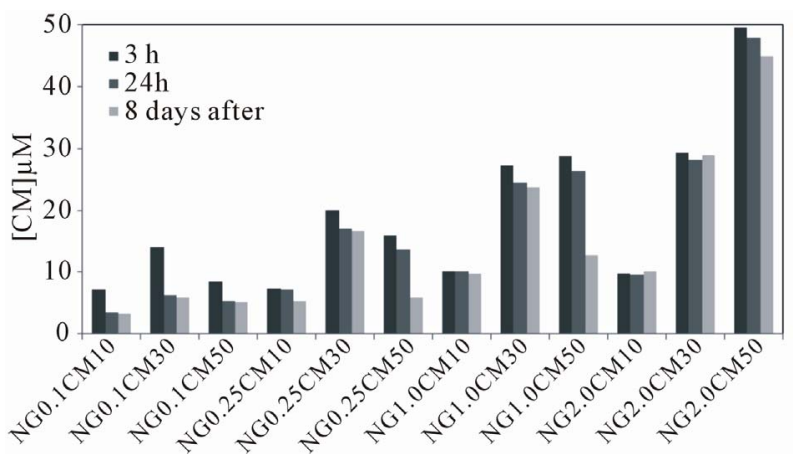

(a)

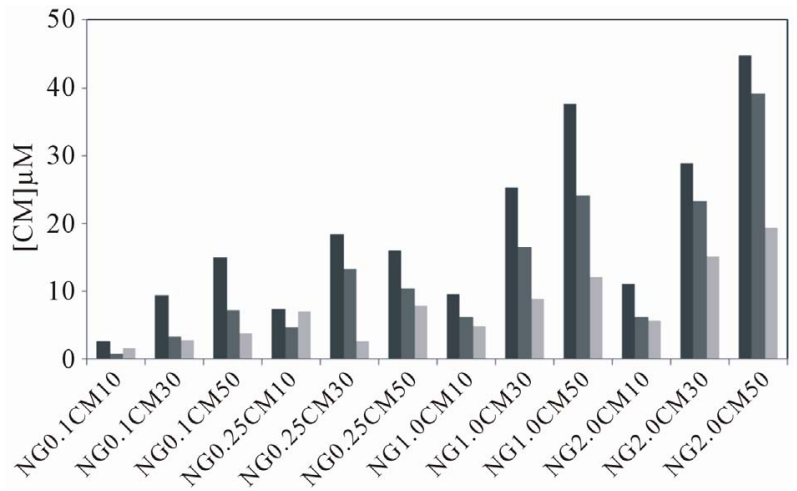

(b)

Figure 2. Curcumin (CM) loading into dextrin nanogel (NG) in (a) aqueous or (b) PBS dispersion of different formulations varying the curcumin $(10,30,50 \mu \mathrm{M})$ and nanogel $(0.1,0.25,1.0$ or $2.0 \mathrm{mg} / \mathrm{mL})$ concentrations, $3 \mathrm{~h}, 24 \mathrm{~h}$ or 8 days after incorporation.

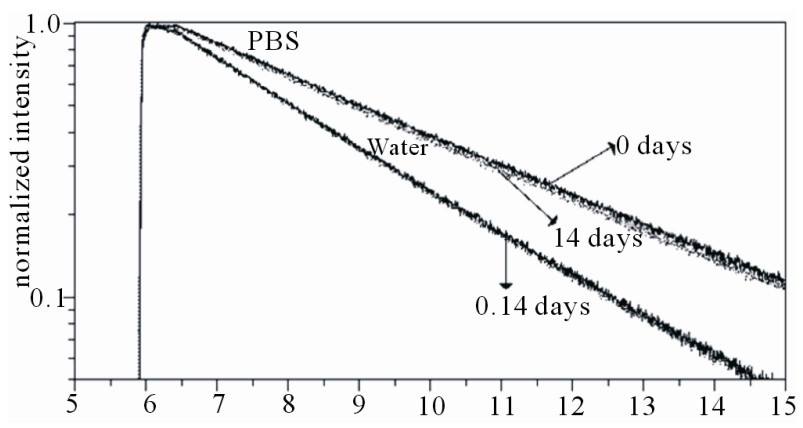

Figure 3. SAMSA-F fluorescence decays for nanogel dispersed in distilled water or in PBS, up to 14 days.

the open system from inside the dialysis membrane) and the soluble drug was quantified spectrophotometrically, after centrifugation (supernatant) to remove the unload curcumin. Therefore, only the entrapped curcumin is quantified.

Figure 4 shows the curcumin concentration along time in closed and open systems. The results obtained for closed system (Figure 4(a)) show that curcumin concentration remains constant, up to 24 hours.

Using dialysis membrane method, a fast transfer of 


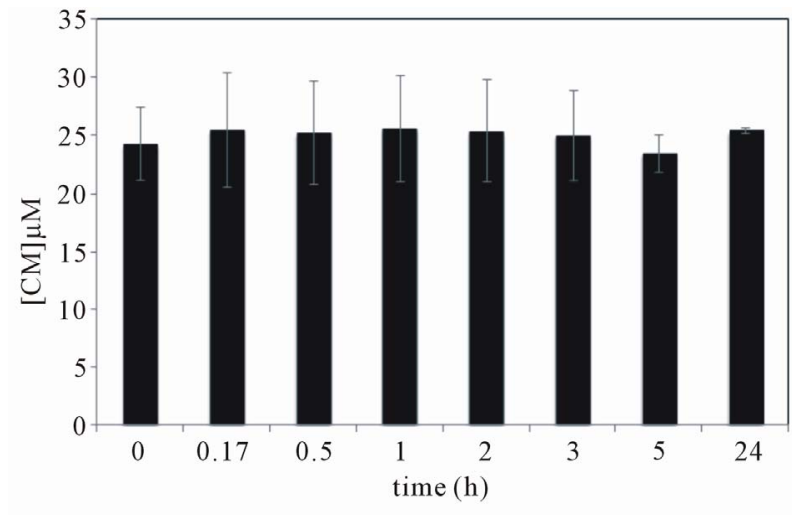

(a)

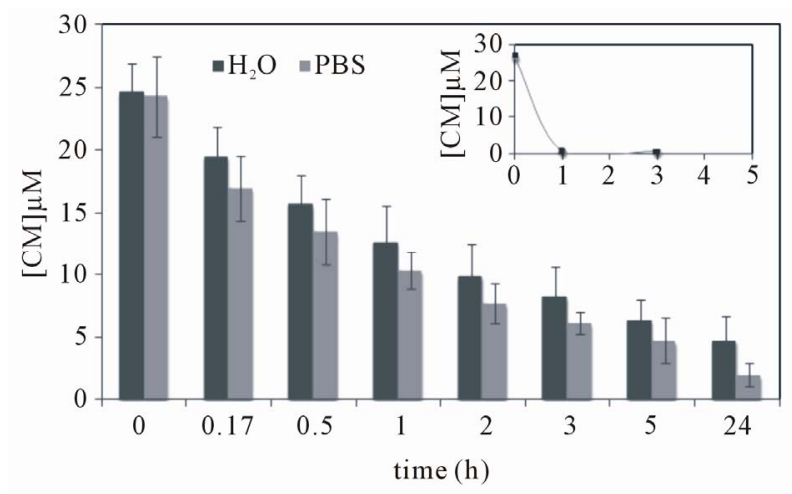

(b)

Figure 4. In vitro curcumin release from dextrin nanogel (1.0 mg/mL) using (a) closed system or (b) sink conditions. In the later case, both distilled water or PBS solution were used during dialysis; a control assay was carried out using curcumin solubilised in ethanol under the same consitions (inset).

curcumin (inset Figure 4(b)) solubilized in ethanol, across the dialysis bag provided evidence for the presence of sink conditions under the experimental set up. Namely, after $1 \mathrm{~h}$ of incubation, $97 \%$ of soluble curcumin was removed of the dialysis bag. Using these conditions (Figure 4(b)), a sustained release of curcumin loaded into nanogel was observed for either release mediums. After 1 and $5 \mathrm{~h}$ of incubation, the fractions of curcumin remaining soluble were respectively $52 \%$ and $26 \%$ using distilled water and $43 \%$ and $19 \%$ for the PBS solution.

The nanogel size distribution was evaluated at the end of dialysis process. The $\mathrm{z}$-average value remained constant during the dialysis process against water $(74.7 \mathrm{~nm})$ and slightly increased when using PBS solution (103 $\mathrm{nm})$.

\subsection{Cytotoxicity Assay}

To assess the biological activity of curcumin in the nanogel formulation, HeLa cells were exposed to culture medium supplemented with nanogel, free curcumin (in etha- nol) or curcumin-loaded nanogel (in distilled water) and cell viability determined at 24 and $48 \mathrm{~h}$ (Figure 5).

Whereas empty nanogel had no effect on cell viability, curcumin-loaded nanogel inhibited cancer cell growth, as effectively as free curcumin.

\section{Discussion}

The possibility of enhancing curcumin's solubility using dextrin nanogel as nanocarrier was investigated in this work. The colloidal stability of dextrin nanogel (in distilled water or PBS solution) has been previously assessed using dexC $\mathrm{C}_{16}$ with a higher degree of substitution (DS $\mathrm{D}_{\mathrm{C} 16} 8.7 \%$ vs $6.0 \%$ in this work), up to 7 days. High stability was observed in both water and PBS solutions, since no aggregates were detected and a low polydispersity index was conserved. As reported, the more substituted polymer forms more densely packed hydrophobic domains, such that the colloidal stability of nanogel is increased [10].

In this work, the stability of dextrin nanogel $\left(\mathrm{DS}_{\mathrm{C} 16}\right.$ $6.0 \%$ ), in distilled water or PBS solution, was evaluated, up to 12 days. A general conclusion may be drawn from the results obtained: the nanogel prepared in a saline solution (buffer) present a gradual size increase along with the incubation period, apparently associated to a swelling process, when compared to those dispersed in water. The effect of salts on lipoprotein (pig serum low density lipoprotein) structure, which is primarily maintained by hydrophobic interactions, has been reported in the literature [11]. The authors concluded that the choice of salt as the co-solvent in the studies of proteins, enzymes, and lipoproteins is of crucial importance. In general, hydrophobic force decreases with increasing $\mathrm{NaCl}$ concentrations [12]. Our group has previously reported that nanogel has a slightly higher size when prepared in the presence of a salt or urea (irrespective of the concentration) [10]. Thus, salts seem to effectively affect the hydrophobic interactions inducing the nanogel to "swell", although the interaction mechanism is not object of study in this work. Indeed, the size increase is not dramatic and is gradual along time. The curcumin-loaded nanogel was

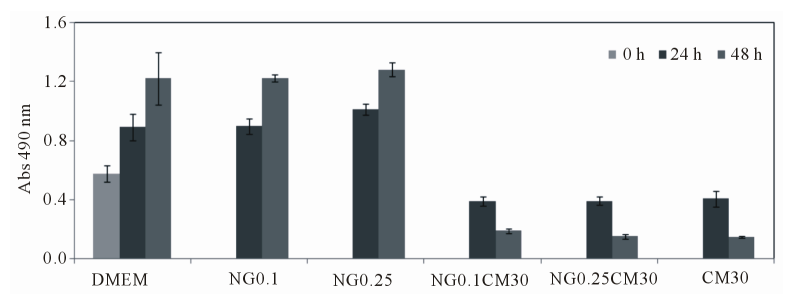

Figure 5. Cell viability of HeLa cells after incubation with different nanogel (NG) concentrations, curcumin-loaded nanogel or unload curcumin (CM). Empty nanogel was used to test the cytotoxicity of the nanocarrier. 
evaluated in this work for loading efficiency and stability. Drug-loaded nanogel should be able to retain the drug enough time as to reach their target. The results of curcumin loading in aqueous solution (Figure 2(a)) shows that for a constant nanogel concentration, the higher curcumin concentration results in lower stability over time. For instance, for the highest nanogel concentration studied $(2.0 \mathrm{mg} / \mathrm{mL}), 10$ or $30 \mu \mathrm{M}$ of curcumin remain stable until 8 days. The lower curcumin concentration tested $(10 \mu \mathrm{M})$ is stabilized by a nanogel concentration of 0.25 $\mathrm{mg} / \mathrm{mL}$. Increasing the curcumin concentration to $30 \mu \mathrm{M}$, a nanogel concentration at least of $1.0 \mathrm{mg} / \mathrm{mL}$ is required. Thus, as could be expected, there is an optimal proportion of nanogel/curcumin that allows a stable formulation. Curcumin loading in PBS (Figure 2(b)) reveals that it is not possible to stabilize curcumin on nanogel, for longer periods, when PBS is used as dispersion medium.

The fluorescence study with SAMSA-F labeled nanogel reveals a faster decay of SAMSA-F average life time, observed in PBS (compared to distilled water), with aging time. This result can be explained by assuming an alteration of the SAMSA-F environment with time from an initially more hydrophobic to a more hydrophilic pocket, as nanogel enlargement would lead to a possibly increased exposure of SAMSA-F to water resulting in a decreased decay time.

Different methods for the evaluation of the release profiles from nanoparticulate systems have been reported. Open [13] and closed systems [14] have been described. In the former case (open system or sink conditions), dialysis membranes are often used. As for closed systems, loaded-nanocarriers are incubated, enclosed in a flask, under agitation. In the present work, the release study of curcumin from nanogel was carried out using both approaches. The results obtained for closed systems show that curcumin remain stable within nanogel, up to 24 hours, confirming the high stability of the formulation in the conditions used. In our opinion, this method assesses curcumin stability instead of curcumin release. Using the dialysis membrane method, a fast transfer of soluble curcumin (ethanol used as solvent) across the dialysis bag provide evidence of sink conditions. Using this method, a sustained release of curcumin was observed for either release mediums (distilled water or PBS). The release profile without initial burst indicates the absence of curcumin adsorbed on the nanogel surface [15]. It is well-known that drug release is affected by nanoparticle size. Smaller particles have a larger surface-to-volume ratio; therefore, most of the drug associated with small particles would be at or near the particle surface, leading to faster drug release. In contrast, larger particles have large cores, which allow more drug encapsulation per particle, providing a slower release. Thus, control of particle size provides a means of tuning the drug release rates. In the current case, the fairly low size of the nanogel may explain the complete release of curcumin in a 24 $\mathrm{h}$ timeframe. The observed profile indicate dextrin nanogel as a suitable carrier for curcumin, slowing down its release when compared with the profile observed for unload curcumin (solubilised in ethanol). The kinetics of curcumin release are compatible with an effective concentration of the carrier in the targeted tissue, followed by a continuous release of curcumin for several hours, a formulation that may have clinical relevance. It must be remarked that, due to the well characterized chemical instability of curcumin, the release over longer time frames becomes useless.

An evaluation of the cytotoxicity of nanogel was carried out in a previous study, using murine bone marrow-derived macrophages: no significant toxicity was found up to concentrations of $1.0 \mathrm{mg} / \mathrm{mL}$ [16]. In this work, the cytotoxicity of the nanogel and its curcumin formulations were evaluated with HeLa cells. In the literature, cytotoxicity studies of HeLa cells showed that the $\mathrm{IC}_{50}$ of free curcumin and complexed curcumin on bovine casein micelles was 14.85 and $12.69 \mu \mathrm{M}$, respecttively. The loaded curcumin has showed a cytotoxic effect that was comparable to that of free curcumin [17]. In this study, as control, Hela cells were kept in the culture medium without any treatment. Nanogel concentration used as a carrier $(0.1$ and $0.25 \mathrm{mg} / \mathrm{mL})$ does not induce cell death up to $24 \mathrm{~h}$, indeed the number of viable cells in contact with nanogel is similar to the control. When treated with curcumin, cells react to loaded curcumin (irrespective of the nanogel concentration) as effectively as free curcumin. The benefit of curcumin load into nanogel is to overcome the limited bioavailability after in vivo administration, without any organic solvent use.

In conclusion, dextrin nanogel served as an effective "nanocarrier" for the formulation of lipophilic curcumin by increasing its water solubility, improving its stability, and controlling its release profile. Incorporation of curcumin into nanogel did not compromise its cytotoxicity as demonstrated with HeLa cell line. Therefore, dextrin nanogel holds promise as drug delivery system for the development of an injectable formulation of curcumin as anti-cancer agent for the treatment of human cancers. The small size of this system $(<100 \mathrm{~nm})$ can be advantageous for passive targeting of tumor tissues by the EPR effect, after intravenous administration. Regarding formulation stability of nanogel, it must be dispersed in water to guarantee suitable property maintenance during storage.

\section{Acknowledgements}

This research was supported by Fundação para a Ciência e a Tecnologia under grant SFRH/BD/22242/2005. 


\section{REFERENCES}

[1] A. Goel, A. B. Kunnumakkara and B. B. Aggarwal, "Curcumin as 'Curecumin': From Kitchen to Clinic," Biochemical Pharmacology, Vol. 75, No. 4, 2008, pp. 787809. doi:10.1016/j.bcp.2007.08.016

[2] M. Lopez-Lazaro, "Anticancer and Carcinogenic Properties of Curcumin: Considerations for Its Clinical Development as a Cancer Chemopreventive and Chemotherapeutic Agent," Molecular Nutrition \& Food Research, Vol. 52, No. S1, 2008, pp. S103-S127. doi:10.1002/mnfr.200700238

[3] E. Tourkina, P. Gooz, J. C. Oates, A. Ludwicka-Bradley, R. M. Silver and S. Hoffman, "Curcumin-Induced Apoptosis in Scleroderma Lung Fibroblasts: Role of Protein Kinase C Epsilon," American Journal of Respiratory Cell and Molecular Biology, Vol. 31, No. 1, 2004, pp. 28-35. doi:10.1165/rcmb.2003-0354OC

[4] A. Kunwar, A. Barik, R. Pandey and K. I. Priyadarsini, "Transport of Liposomal and Albumin Loaded Curcumin to Living Cells: An Absorption and Fluorescence Spectroscopic Study," Biochimica et Biophysica Acta, Vol. 1760, No. 10, 2006, pp. 1513-1520. doi:10.1016/i.bbagen.2006.06.012

[5] G. P. Lim, T. Chu, F. Yang, W. Beech, S. A. Frautschy and G. M. Cole, "The Curry Spice Curcumin Reduces Oxidative Damage and Amyloid Pathology in an Alzheimer Transgenic Mouse," The Journal of Neuroscience, Vol. 21, No. 21, 2001, pp. 8370-8377.

[6] M. T. Huang, T. Lysz, T. Ferraro, T. F. Abidi, J. D. Laskin and A. H. Conney, "Inhibitory Effects of Curcumin on in Vitro Lipoxygenase and Cyclooxygenase Activities in Mouse Epidermis," Cancer Research, Vol. 51, No. 3, 1991, pp. 813-819.

[7] M. M. Chan, H. I. Huang, M. R. Fenton and D. Fong, "In Vivo Inhibition of Nitric Oxide Synthase Gene Expression by Curcumin, a Cancer Preventive Natural Product with Anti-Inflammatory Properties," Biochemical Pharmacology, Vol. 55, No. 12, 1998, pp. 1955-1962. doi:10.1016/S0006-2952(98)00114-2

[8] G. Shoba, D. Joy, T. Joseph, M. Majeed, R. Rajendran and P. S. Srinivas, "Influence of Piperine on the Pharmacokinetics of Curcumin in Animals and Human Volunteers," Planta Medica, Vol. 64, No. 4, 1998, pp. 353-356. doi:10.1055/s-2006-957450

[9] C. Goncalves, J. A. Martins and F. M. Gama, "Self-As- sembled Nanoparticles of Dextrin Substituted with Hexadecanethiol," Biomacromolecules, Vol. 8, No. 2, 2007, pp. 392-398. doi:10.1021/bm060993e

[10] C. Gonçalves and F. M. Gama, "Characterization of the Self-Assembly Process of hydrophobically Modified Dextrin," European Polymer Journal, Vol. 44, No. 11, 2008, pp. 3529-3534. doi:10.1016/j.eurpolymj.2008.08.034

[11] M. Janado, Y. Yano, H. Nishida and T. Nishida, "Effect of Salt on the Intermolecular and Intramolecular Hydrophobic Interactions of Macromolecules," Journal of Solution Chemistry, Vol. 15, No. 10, 1986, pp. 839-850. doi:10.1007/BF00646091

[12] L. G. Wang and R. H. Yoon, "Hydrophobic Forces in the Foam Films Stabilized by Sodium Dodecyl Sulfate: Effect of Electrolyte," Langmuir, Vol. 20, No. 26, 2004, pp. 11457-11464. doi:10.1021/la048672g

[13] J. Shaikh, D. D. Ankola, V. Beniwal, D. Singh and M. N. Kumar, "Nanoparticle Encapsulation Improves Oral Bioavailability of Curcumin by at Least 9-Fold When Compared to Curcumin Administered with Piperine as Absorption Enhancer," European Journal of Pharmaceutical Sciences, Vol. 37, No. 3-4, 2009, pp. 223-230. doi:10.1016/j.ejps.2009.02.019

[14] R. K. Das, N. Kasoju and U. Bora, "Encapsulation of Curcumin in Alginate-Chitosan-Pluronic Composite Nanoparticles for Delivery to Cancer Cells," Nanomedicine, Vol. 6, No. 1, 2009, pp. 153-160. doi:10.1016/j.nano.2009.05.009

[15] R. Mulik, K. Mahadik and A. Paradkar, "Development of Curcuminoids Loaded Poly(Butyl) Cyanoacrylate Nanoparticles: Physicochemical Characterization and Stability study," European Journal of Pharmaceutical Sciences, Vol. 37, No. 3-4, 2009, pp. 395-404. doi:10.1016/j.ejps.2009.03.009

[16] C. Gonçalves, E. Torrado, T. Martins, P. Pereira, J. Pedrosa and M. Gama, "Dextrin Nanoparticles: Studies on the Interaction with Murine Macrophages and Blood Clearance," Colloids and Surfaces B: Biointerfaces, Vol. 75, No. 2, 2010, pp. 483-489. doi:10.1016/j.colsurfb.2009.09.024

[17] A. Sahu, N. Kasoju and U. Bora, "Fluorescence Study of the Curcumin-Casein Micelle Complexation and Its Application as a Drug Nanocarrier to Cancer Cells," Biomacromolecules, Vol. 9, No. 10, 2008, pp. 2905-2912. doi:10.1021/bm800683f 